Ann. Sci. forest., 1977, 34 (4), 285-292.

\title{
Etude de la variabilité spécifique et infraspécifique de la structure juvénile du bois de chêne à l'aide d'un analyseur d'images
}

\author{
H. POLGE *, M. LEMOINE ** ef ÉLIANE DERET * \\ * Station de Recherches sur la Qualité des Bois, \\ Centre National de Recherches Forestières, I.N.R.A., \\ Champenoux, 54280 Seichamps \\ ** Station d'Amélioration des Arbres Forestiers, \\ Centre de Recherches Forestières d'Orléans, I.N.R.A., \\ Ardon, 45160 Olivet
}

\section{Résumé}

Un analyseur d'images a été utilisé pour mesurer le diamètre, l'épaisseur de l'écorce et le pourcentage de fibres de semis de chêne de 4 ans prélevés dans une plantation comparative de provenances.

Des différences significatives ont pu être mises en évidence pour le diamètre et le pourcentage de fibres, mais malheureusement la provenance qui a la croissance la plus rapide (Amance) est également celle dont le bois contient le pourcentage de fibres le plus élevé.

De même, à l'intérieur de chaque provenance, la corrélation entre diamètre et pourcentage de fibres est positive et significative.

Au vu de cette expérience, l'analyseur d'images apparaît comme un outil efficace pour quantifier les données relatives à l'anatomie du bois.

Les spécialistes de l'amélioration des arbres forestiers souhaitent toujours pouvoir déterminer le plus rapidement possible les performances des jeunes plants de leurs tests de descendances ou de provenances; la connaissance précoce des divers critères de croissance ou de qualité leur permet en effet, tout au moins lorsque les corrélations juvénile-adulte sont étroites comme c'est le cas pour la plupart des caractéristiques du bois, de rendre plus rapide et plus sûr le travail de sélection.

Le but de l'expérience dont il est rendu compte ici était de savoir s'il était possible de comparer, dès i'âge de 4 ans, la qualité du bois de 4 provenances françaises de chêne : 3 de chêne rouvre (Bercé, Tronçais, Darney), 1 de chêne pédonculé (Amance).

\section{Matériel et méthode}

\subsection{Echantillonnage}

Le dispositif expérimental, planté il y a 4 ans, comprend 50 blocs et 10 plants par parcelle unitaire. A la fin de la première quinzaine de mars 1976, on a recépé la moitié des blocs, en alternant les blocs recépés et laissés intacts, afin d'étudier l'influence du recépage sur la forme des arbres. Dans chaque provenance et dans 
chaque bloc, on a choisi au hasard deux plants sur lesquels on a prélevé $5 \mathrm{~cm}$ de tige à partir du collet. On a donc prélevé 50 échantillons par provenance.

\subsection{Choix de la méthode}

Le critère de qualité du bois le plus couramment utilisé est la densité ; sa détermination se heurtait cependant, au cas particulier, à un certain nombre de difficultés :

La méthode par double pesée (poids saturé et poids anhydre) ne pouvait guère être envisagée avec des échantillons dont certains n'atteignaient même pas $3 \mathrm{~mm}$ de diamètre sur écorce ; il aurait fallu, pour obtenir des valeurs de densité valables, les mesurer sur des fragments de tiges de grande longueur, ce qui aurait rendu leur saturation intégrale incertaine.

La détermination des volumes au voluménomètre à mercure aurait été tout aussi aléatoire pour un feuillu à zone poreuse où le risque de pénétration du mercure dans les gros vaisseaux du bois de printemps n'est pas négligeable.

Enfin, la partie inférieure des tiges dont nous disposions comportait de nombreuses petites branches et également des nœuds cachés qui risquaient de fausser les valeurs de densité.

Le recours à des mesures anatomiques semblait donc s'imposer mais il n'apparaissait pas possible d'y procéder sur un grand nombre d'échantillons en utilisant les méthodes traditionnelles : dessins à la chambre claire ou sur l'écran du microscope

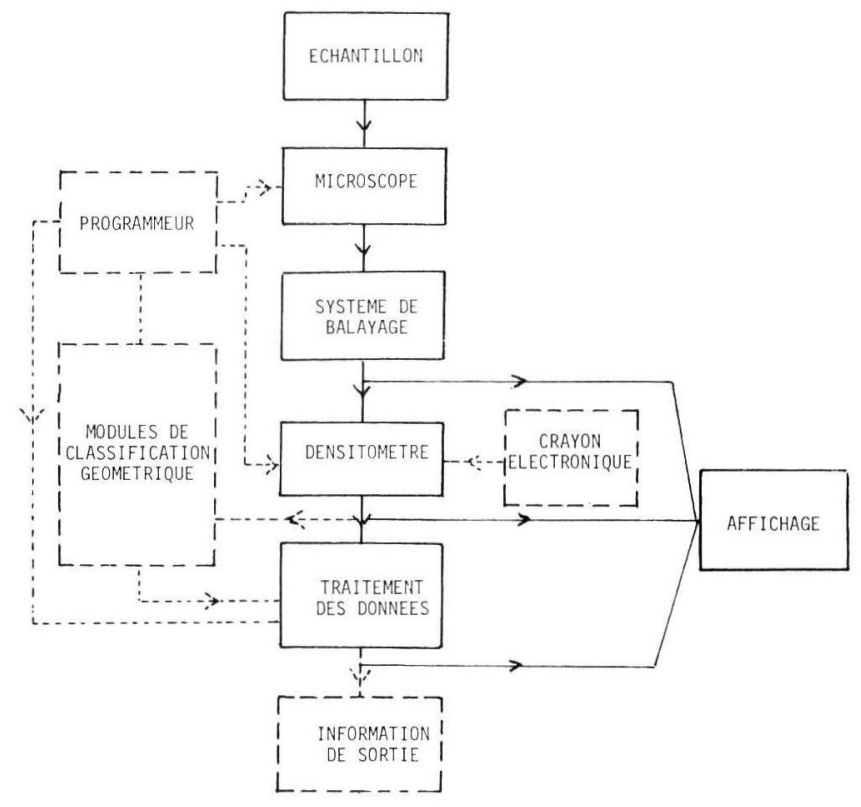

FIG. 1. - Schéma d'un analyseur d'images.

En traits pleins : équipement de base.

En traits interrompus : modules en option.

Diagram of an image analyser

Full lines : basic équipment.

Dashed lines : optional modules. 
de projection, et calculs très laborieux des surfaces occupées par les différents types d'éléments anatomiques.

Il était donc tentant de profiter de cette expérience pour faire l'essai d'un appareil automatique d'analyse d'images qui, mis au point initialement pour des études de métallographie, semblait pouvoir être utilisé avec profit pour résoudre les problèmes d'anatomie quantitative du bois qui se trouvaient posés.

\section{3. - Description d'un analyseur d'images (fig. 1 et 2)}

L'appareil dont nous avons disposé, un quantimet 720 D, aimablement prêté par son constructeur : la société IMANCO, comprend 4 parties :

- le système d'entrée de l'image qui peut être un microscope ordinaire disposant ou non d'un déplacement automatique pas à pas de la platine, ou bien un épidiascope,

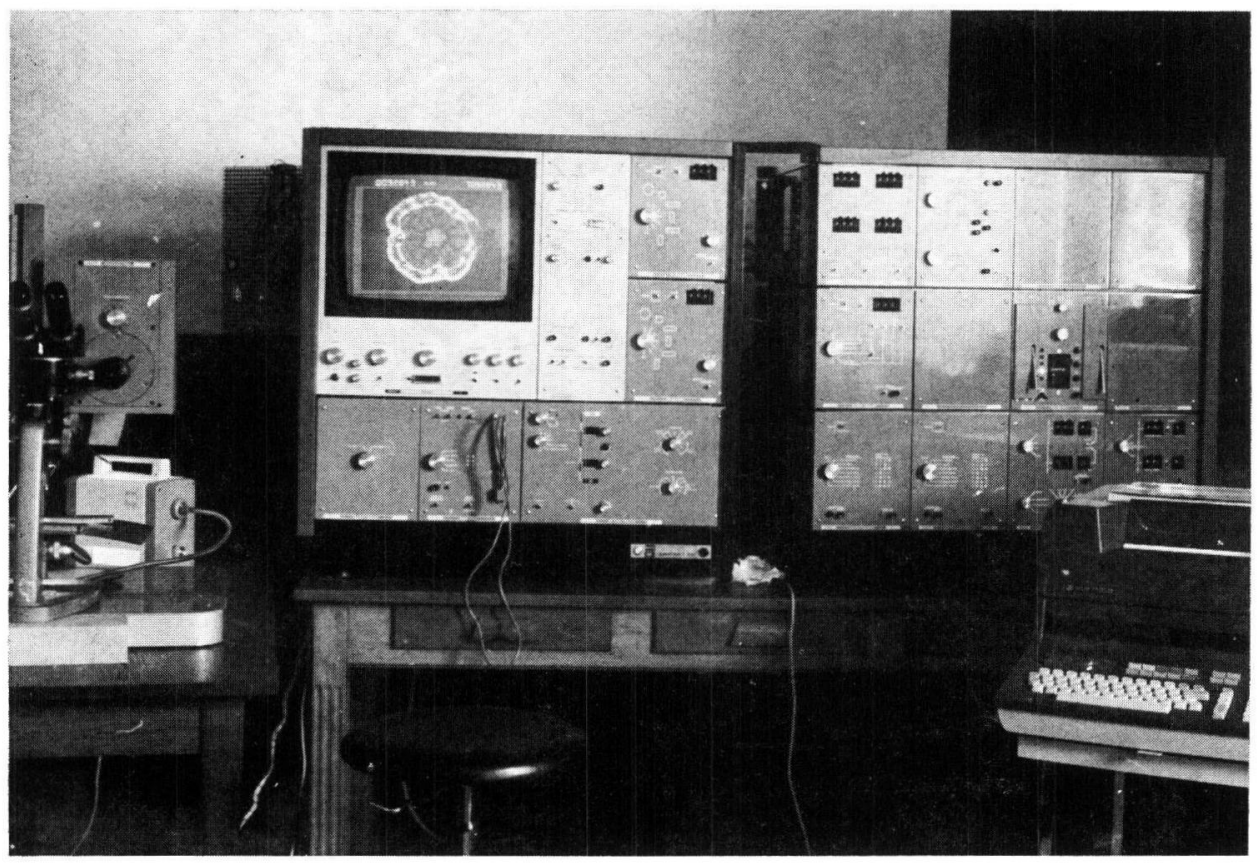

De gauche à droite :

FIG. 2. - Analyseur d'images.

- la caméra de télévision montée sur microscope.

- l'unité centrale comportant notamment en haut, à gauche, l'écran de télévision sur lequel on peut voir l'image non seuillée d'une rondelle de chêne et à la partie inférieure le module de correction de l'image (crayon électronique).

- les divers modules de classification géométrique.

- le calculateur programmable.

From the left to the right :

Image analyser.

- The vidicon scanner connected to a microscope.

- The central unit.

- The different modules of geometrical classification.

- The calculator. 
- une unité centrale regroupant:

- une caméra de télévision munie d'un système de balayage à 720 lignes à haut pouvoir de résolution qui convertit l'image optique en une image électrique, le signal VIDEO produit par l'analyseur étant numérisé spatialement dans une matrice fixe de 605000 points-image,

- un module de séparation des niveaux de gris, véritable densitomètre qui détermine l'absorption de lumière sur chaque point-image et assigne à chacun d'eux un code binaire équivalant au logarithme de l'absorption optique qui les caractérise.

- une unité de traitement des données dans laquelle la contribution en densité optique de chaque point de l'image à l'intérieur d'une aire choisie est calculée de façon à obtenir la densité totale intégrée de cette surface.

- des systèmes d'acquisition de données que l'on peut connecter à l'analyseur d'images par l'intermédiaire d'interfaces appropriés (simples télétypes ou calculatrices plus ou moins performantes) et qui peuvent notamment fournir des diagrammes de fréquence des particules classées suivant leur surface, leur périmètre, leur plus grande longueur, leur plus grande largeur, etc...

Le procédé le plus simple de sélection des divers composants de l'image est basé sur les niveaux de gris, l'appareil indiquant les surfaces (en nombre de points-image) dont la densité optique est supérieure ou inférieure à un seuil déterminé, ou encore comprise entre deux seuils de densité, et ceci, soit sur la totalité du champ, soit sur une fraction seulement de celui-ci préalablement délimitée.

II est également possible d'employer d'autres critères de sélection, et notamment des critères géométriques comme la longueur, la surface ou le périmètre des particules.

Un module de correction bi-dimensionnel permet une séparation automatique des images élémentaires et l'élimination de détails parasites par le jeu successif de dilatations et d'érosions.

Enfin, grâce à un autre module, il est possible de corriger manuellement les imaçe; en délimitant, à l'aide d'un crayon électronique, des surfaces qui seront seules analysées ou, au contraire, qui sont à supprimer, de séparer des particules qui, dans l'analyse automatique, risqueraient d'être à tort comptées comme une seule ou, opération inverse, de réunir deux portions d'une même particule qui pourraient à tort être comptées séparément, de compléter un contour qui n'apparaîtrait pas de façon nette sur l'écran, ou au contraire de supprimer des appendices ne faisant pas partie intégrante des particules.

\section{4. - Mode opératoire}

On aurait pu penser analyser à l'aide du quantimet des coupes microscopiques des tiges de chêne à étudier ; en dehors des délais très importants qu'exige la confection des coupes (vidage des ccllules, coloration des membranes, fixation, montage des échantillons), ceci n'a pas été fait car il est extrêmement difficile, même avec un excellent microtome, d'obtenir, à partir de tiges de faible diamètre, des coupes d'épaisseur rigoureusement uniforme; des différences d'épaisseur de l'ordre de 40 p. 100 (par exemple de $10 \mu$ sur 25 ) ne sont pas à exclure, et entraînent, lors de leur exploration par une caméra de télévision noir et blanc, des différences de noircissement qui risquent de fausser l'expérience. 
On a jugé préférable de soumettre à l'analyse l'image radiographique de sections de tiges de $0,8 \mathrm{~mm}$ d'épaisseur débitées à l'aide de scies-fraises jumelées (F. Thiercelin et J. R. Perrin, 1972). La précision du sciage est de l'ordre de 1/100e de $\mathrm{mm}$ et l'uniformité d'épaisseur traversée par le rayonnement est donc satisfaisante.

Un bon contraste des images a été obtenu en utilisant les conditions d'exposition suivantes :

- distance source-film : $2,50 \mathrm{~m}$;

- tension accélératrice : $8 \mathrm{kV}$;

- intensité du flux électronique : $18 \mathrm{~mA}$;

- durée d'exposition : $48 \mathrm{~h}$.

L'analyseur d'images a fourni 3 données brutes :

- la surface totale sur écorce de chaque rondelle obtenue automatiquement par sélection à partir des blancs, puisque les échantillons se détachaient en plus clair sur le fond de la radiographie,

- la surface sous écorce qui, n'ayant pas pu se faire automatiquement en raison de la très grande variabilité de la densité des écorces et de la présence possible de zones de même noircissement dans celles-ci ct dans le bois, a été lue après délimitation des deux parties à l'aide du crayon électronique,

- la surface cumulée des fibres et des rayons ligneux, obtenue par une sélection de gris qui a laissé de côté les vaisseaux et le parenchyme périvasculaire.

Connaissant l'équivalence grandeur vraie - nombre de points-image, il a été possible de passer des surfaces sous et sur écorce aux diamètres moyens correspondants.

\section{2. - Résultats}

\section{1. - Diamètre sur écorce}

Les valeurs moyennes et les coefficients de variation sont les suivants :

\begin{tabular}{|c|c|c|}
\hline & Moyenne & Coefficient de variation \\
\hline $\begin{array}{l}\text { Bercé } \ldots \ldots \ldots \ldots \ldots \ldots \\
\text { Darney } \ldots \ldots \ldots \ldots \ldots \ldots \ldots \ldots \\
\text { Tronçais } \ldots \ldots \ldots \ldots \ldots \ldots \ldots \ldots \\
\text { Amance } \ldots \ldots \ldots \ldots \ldots \ldots \ldots \\
\text { Ensemble } \ldots \ldots \ldots \ldots \ldots \ldots\end{array}$ & $\begin{array}{l}8,64 \mathrm{~mm} \\
7,90 \mathrm{~mm} \\
8,00 \mathrm{~mm} \\
9,58 \mathrm{~mm} \\
8,53 \mathrm{~mm}\end{array}$ & $\begin{array}{l}41,3 \text { p. } 100 \\
47,0 \text { p. } 100 \\
34,9 \text { p. } 100 \\
36,3 \text { p. } 100 \\
40,4 \text { p. } 100\end{array}$ \\
\hline
\end{tabular}

Les résultats de l'analyse de variance correspondante sont donnés ci-dessous :

\begin{tabular}{lrrr}
\hline Source de variation & Somme des carrés & DDL & Carré moyen \\
\hline Provenance $\ldots \ldots \ldots \ldots$ & 89,138 & 3 & 29,712 \\
Erreur $\ldots \ldots \ldots \ldots \ldots$ & 2260,033 & 195 & 11,590 \\
Total $\ldots \ldots \ldots \ldots \ldots$ & 2349,171 & 198 & \\
\hline
\end{tabular}

La valeur du test $F$ obtenu est extrêmement voisine du seuil de signification à 5 p. 100 qui, pour 3 et 195 degrés de liberté, s'élève à 2,60. 
Les résultats des comparaisons de moyennes se présentent comme suit :

Darney Tronçais Bercé Amance

\section{2. - Epaisseur de l'écorce}

Bien qu'il ne s'agisse là, ni d'un critère de qualité du bois, ni d'un critère de croissance à proprement parler, elle a été calculée à partir des données brutes fournies par l'analyseur dans le seul but d'utiliser l'ensemble des résultats pour analyser la variabilité infraspécifique et mettre en évidence éventuellement un critère de différenciation des provenances.

Les valeurs moyennes et les coefficients de variation sont les suivants :

\begin{tabular}{|c|c|c|}
\hline & Moyenne & Coefficient de variation \\
\hline 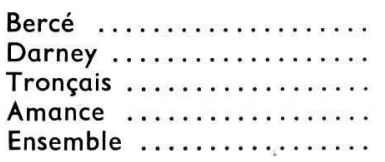 & $\begin{array}{l}0,84 \mathrm{~mm} \\
0,75 \mathrm{~mm} \\
0,85 \mathrm{~mm} \\
0,89 \mathrm{~mm} \\
0,83 \mathrm{~mm}\end{array}$ & $\begin{array}{l}42,2 \text { p. } 100 \\
46,0 \text { p. } 100 \\
40,2 \text { p. } 100 \\
40,0 \text { p. } 100 \\
41,3 \text { p. } 100\end{array}$ \\
\hline
\end{tabular}

Les résultats de l'analyse de variance correspondante sont donnés ci-dessous :

\begin{tabular}{lrrr}
\hline Source de variation & Somme des carrés & DDL & Carré moyen \\
\hline Provenance $\ldots \ldots \ldots \ldots$ & 0,57078 & 3 & 0,19026 \\
Erreur $\ldots \ldots \ldots \ldots \ldots$ & 22,61350 & 194 & 1,63 \\
Total $\ldots \ldots \ldots \ldots \ldots \ldots$ & 23,18428 & 197 & 0,11656 \\
\end{tabular}

Il n'y a donc pas globalement de différence entre lis provenances au point de vue de l'épaisseur de l'écorce.

\section{3. - Pourcentage de fibres}

C'est du point de vue de la qualité du bois de chêne la caractéristique la plus intéressante à étudier et on peut dire en première analyse que les provenances seront d'autant plus aptes à fournir des produits de choix, et notamment du tranchage, que ce pourcentage sera plus réduit.

Les valeurs moyennes et les coefficients de variation sont les suivants :

\begin{tabular}{lll}
\hline & Moyenne & Ecart-type \\
\hline Bercé $\ldots \ldots \ldots \ldots \ldots \ldots$ & 38,59 p. 100 & 16,10 p. 100 \\
Darney $\ldots \ldots \ldots \ldots \ldots$ & 35,13 p. 100 & 16,54 p. 100 \\
Tronçais $\ldots \ldots \ldots \ldots \ldots$ & 32,25 p. 100 & 20,75 p. 100 \\
Amance $\ldots \ldots \ldots \ldots \ldots$ & 46,19 p. 100 & 11,29 p. 100 \\
Ensemble $\ldots \ldots \ldots \ldots$ & 38,04 p. 100 & 17,25 p. 100 \\
\hline
\end{tabular}


Les résultats de l'analyse de variance correspondante sont donnés ci-dessous :

\begin{tabular}{|c|c|c|c|c|}
\hline Source de variation & Somme des carrés & $\mathrm{DDL}$ & Carré moyen & $\mathrm{F}$ \\
\hline $\begin{array}{l}\text { Provenance }: \ldots \ldots \ldots \ldots \\
\text { Erreur } \ldots \ldots \ldots \ldots \ldots \ldots \ldots \\
\text { Total } \ldots \ldots \ldots \ldots \ldots \ldots\end{array}$ & $\begin{array}{l}0,5440 \\
5,3202 \\
5,8642\end{array}$ & $\begin{array}{r}3 \\
195 \\
198\end{array}$ & $\begin{array}{l}0,1813 \\
0,0273\end{array}$ & 6,646 \\
\hline
\end{tabular}

Les résultats des comparaisons de moyennes se présentent comme suit :

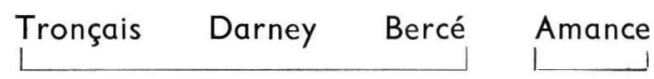

\section{3. - Commentaires}

L'analyseur d'images a permis, à partir de simples radiographies de rondelles minces des tiges-échantillons, de faire apparaître des différences significatives pour deux des trois critères étudiés ; en ce qui concerne le pourcentage de fibres, il est remarquable que la discrimination se produise au niveau de l'espèce puisque la provenance unique de chêne pédonculé se sépare nettement des trois provenances de chêne rouvre. Pour le diamètre sur écorce, on n'a certes pas de différence entre Amance et Bercé, mais les 3 provenances de chêne rouvre ont une croissance inférieure à la provenance de pédonculé et la différence demeure significative pour deux d'entre elles.

A condition de parvenir à améliorer la qualité, et en particulier l'uniformité d'épaisseur des coupes microscopiques, il n'est guère douteux qu'un tel appareil rendrait possibles des analyses beaucoup plus fines, permettant notamment de séparer les uns des autres tous les types d'éléments anatomiques (vaisseaux, fibres, parenchyme périvasculaire et parenchyme des rayons ligneux) et aussi d'obtenir de très utiles diagrammes de répartition par dimensions des plus gros d'entre eux : les vaisseaux, ainsi que cela a d'ailleurs été fait, à l'occasion du prêt de cet appareil, sur des coupes de chêne adulte.

En ce qui concerne les résultats, on peut tout d'abord noter une certaine opposition entre les provenances au point de vue de la variabilité pour les trois caractères étudiés, la provenance Tronçais apparaissant comme relativement homogène pour le diamètre sur écorce et pour l'épaisseur de l'écorce et étant en revanche le plus variable pour le pourcentage de fibres.

On doit aussi observer que le classement des provenances au point de vue diamètre est à peu près le même que celui obtenu pour le pourcentage de fibres, la provenance de chêne pédonculé étant en particulier à la fois caractérisée par une bonne croissance et par un très fort pourcentage de fibres.

Cette liaison défavorable au niveau inter-provenance se vérifie malheureusement aussi à l'intérieur des provenances puisque les coefficients de corrélation entre diamètre sur écorce et pourcentage de fibres s'élèvent à 0,70 pour la provenance Tronçais, 0,56 pour la provenance Darney, 0,46 pour la provenance Bercé et 0,55 pour la provenance Amance, le coefficient global calculé sur l'ensemble des 198 échantillons étant quant à lui de 0,57 ; tous ces coefficients sont significatifs au seuil de 1 p. 1000. 
On peut cependant noter que cette opposition entre croissance rapide et bonne qualité du bois ne s'est retrouvée ni en forêt de Tronçais (H. Polge, R. Keller, 1973), ni en forêt de Bride ou de St Jean (F. Courtoisier, 1976), lorsque l'on a comparé entre eux des chênes adultes d'une même parcelle.

Enfin, bien qu'il faille se garder de toute conclusion prématurée, surtout à un stade aussi jeune, on ne peut pas ne pas observer que les pourcentages de fibres obtenues concordent assez bien avec la réputation des 4 provenances en cause, la provenance Tronçais apparaissant comme celle qui a le bois le plus tendre (même si son pourcentage de fibres n'est pas significativement différent de celui de Darney et de Bercé) et la provenance Amance le bois le plus dur.

\section{Reçu pour publication en avril 1977.}

\section{Remerciements}

M. Thiercelin, Ingénieur à la Station de Recherches sur la Qualité des Bois, a largement contribué à la mise au point des méthodes d'analyse d'images appliquées à l'anatomie du bois ; qu'il trouve ici l'expression de nos remerciements.

\section{Summary}

Study of juvenile oak wood structure specific and infraspecific variability by using an image analysing computer

An electronic image analyser has been used to measure the diameter, the bark thickness and the percentage of fibers on 4 years old oak seedlings within a provenance trial :

Significant differences appear for diameter and for fiber percentages, but unfortunately, the provenance which has the quickiest growth (Amance) also has the highest percentage of fibers.

Similarly, within each provenance the correlation between diameter and fiber percentage is significantly positive. features.

From this experience the image analyser proved to be a fruitful tool for quantifying anatomical

\section{Zusammenfassung}

Untersuchung der spezifischen und infraspezifischen Variabilität der Struktur jungen Eichenholzes an Hand eines Bildanalysators

Ein Bildanalysator wurde zur Messung von folgenden Daten eingesetzt : Durchmesser, Rindendicke und Faseranteil bei 4 Jahre alten Eichensämlingen aus einer Pflanzung zum Abstammungsvergleich. Bezeichnende Unterschiede bezüglich des Durchmessers und des Faseranteils traten dabei in Erscheinung, aber das Holz derjenigen Abstammung mit dem schnellsten Wachstum (Amance) enthält leider auch den höchsten Faseranteil.

Innerhalb einer jeden Abstammung besteht ebenfalls eine positive und bezeichnende Korrelation zwischen dem Durchmesser und dem Faseranteil.

Bei diesem Versuch zeigł sich der Bildanalysator als leistungsfähiges Gerät zur quantitativen Messung von anatomischen Eigenschaften des Holzes.

\section{Références bibliographiques}

COURTOISIER F. Ełude des relations entre stations et qualité du bois de chêne en forêt de Bride et de St Jean. Rapport de stage ENITEF, Station de Recherches sur la Qualité des Bois, C.N.R.F., juillet 1976.

POLGE H., KELLER R. Qualité du bois et largeur d'accroissements en forêt de Tronçais. Annales des Sciences Forestières, 1973, 30, 2, 91-126.

THIERCELIN F., PERRIN J. R. Débit d'échantillons d'épaisseur rigoureusement uniforme à l'aide de scies-fraises jumelées. Annales des Sciences Forestières, 1972, 30, 2, 295-299. 